\title{
On Optimizing Backoff Counter Reservation and Classifying Stations for the IEEE 802.11 Distributed Wireless LANs
}

\author{
Yang Xiao, Senior Member, IEEE, Frank Haizhon Li, Student Member, IEEE, \\ Kui Wu, Member, IEEE, Kin K. Leung, Fellow, IEEE, and Qiang Ni, Member, IEEE
}

\begin{abstract}
In this paper, we propose a novel contention-based protocol called backoff counter reservation and classifying stations (BCR-CS) for the IEEE 802.11 distributed coordination function (DCF). In the proposed scheme, each station has three states: idle, reserved, and contentious. A station is in the idle state if it has no frame ready to transmit. A station is in the reserved state if it has a frame ready to transmit and this frame's backoff counter has been successfully announced through the previous successfully transmitted frame so that other stations know this information. A station is in the contentious state if it has a frame ready to transmit, but this frame's backoff counter has not been successfully announced to other stations. All the stations in the idle state, the reserved state, and the contentious state form an idle group, a reserved group, and a contentious group, respectively. Two backoff schemes are proposed in the BCR-CS protocol based on the number of stations in the contentious group including the optimal pseudo-p-persistent scheme. The proposed schemes are compared with the DCF and the Enhanced Collision Avoidance (ECA) scheme in the literature. Extensive simulations and some analytical analysis are carried out. Our results show that all proposed schemes outperform both the DCF and the ECA, and the BCR-CS with optimal pseudo-p-persistent scheme is the best scheme among the four schemes.
\end{abstract}

Index Terms-IEEE 802.11, medium access control, contention-based, CSMA/CA.

\section{INTRODUCTION}

$\mathrm{T}$ HE IEEE 802.11 Medium Access Control (MAC) employs a mandatory contention-based channel access function called Distributed Coordination Function (DCF), and an optional centrally controlled channel access function called Point Coordination Function (PCF) [1]. The DCF adopts a carrier sense multiple access with collision avoidance (CSMA/CA) with binary exponential backoff. In the DCF a station having a frame to transmit monitors the channel activities until an idle period, equal to a distributed interframe space (DIFS), is detected. After sensing an idle DIFS, the station waits for a random backoff interval before transmitting. The backoff time counter is decremented in terms of slot time as long as the channel is sensed idle. The counter is suspended when a transmission is detected on the channel, and resumes decrementing when the channel is sensed idle again after a DIFS idle time. The station

- Y. Xiao is with the Department of Computer Science, The University of Memphis, Memphis, TN 38152. E-mail: yangxiao@ieee.org.

- F.H. Li is with the Division of Computer Science, University of South Carolina, Upstate, Spartanburg, SC 29303. E-mail: fli@uscupstate.edu.

- K. Wu is with the Department of Computer Science, University of Victoria, Victoria, British Columbia, Canada V8W 3P6.E-mail: wkui@cs.uvic.ca.

- K.K. Leung is with the Electrical and Electronic Engineering and Computing Departments, Imperial College, London SW7 2BT, United Kingdom.E-mail: kkleung@ieee.org.

- Q. Ni is with the Electronic and Computer Engineering, School of Engineering and Design, Brunel University, Uxbridge UB8 3PH, United Kingdom. E-mail: Qiang.Ni@ieee.org.

Manuscript received 27 June 2005; revised 1 Sept. 2005; accepted 22 Sept. 2005; published online 25 May 2006.

Recommended for acceptance by Y. Pan.

For information on obtaining reprints of this article, please send e-mail to: tpds@computer.org, and reference IEEECS Log Number TPDS-0306-0605. transmits its frame when the backoff time reaches zero. At each transmission, the backoff time is uniformly chosen in the range $[0, C W-1]$, where $C W$ is the current backoff window size. At the very first transmission attempt, $C W$ equals to the initial backoff window size $C W_{\min }$. After each unsuccessful transmission, $C W$ is doubled until a maximum backoff window size value $C W_{\max }$ is reached. Once it reaches $C W_{\text {max }}, C W$ shall remain at the value $C W_{\max }$ until it is reset. $C W$ is reset to $C W_{\min }$ after every successful attempt to transmit, or the retransmission counter reaches the retry limit Lretry. In the latter case, the frame will be dropped. After the destination station successfully receives the frame, it transmits an acknowledgment frame (ACK) following a short interframe space (SIFS) time. If the transmitting station does not receive the ACK within a specified ACK Timeout, or it detects the transmission of a different frame on the channel, it schedules the frame retransmission according to the above backoff rules.

There have been many performance studies and enhancements for the DCF [2], [3], [4], [5], [6], [7], [8], [9], [10], [11], [12], [13], [14], [16], [17], [18], [19], [20] reported in the literature. Calì et al. [2] studied an optimization method for a $p$-persistent Wireless LAN (WLAN) MAC, and proposed adaptive backoff algorithms for the $p$-persistent WLAN MAC [3]. Bianchi [4] proposed a simple and accurate analytical model to compute saturation throughput. Ziouva and Antonakopoulos [5] improved Bianchi's model to derive saturation delay. Bing and Subramanianb [6] provided a performance analysis by a quantitative approach. Huang and Chen [7] gave approximate models that account for hidden terminals. Chhaya and Gupta [8] calculated the throughput of CSMA/CA using a simple model with the probabilities of capture and the 
presence of hidden stations. Tay and Chua [9] provided a good approximate model for CSMA/CA. Xiao and Rosdahl [10], [11] identified a throughput upper limit for higher data rates and studied a group transmission and acknowledgement scheme. Leung et al. [12] studied the IEEE 802.11 MAC for outdoor cellular networks. Baldwin et al. [13], [14] proposed a real-time MAC protocol for ad hoc WLANs, in which two concepts are adopted, transmission deadline and a station's next backoff value (BV). The transmission deadline is used for selectively discarding frames which are late for their transmission deadlines at the MAC layer. A station's next $\mathrm{BV}$ is adopted to decrease collisions under a constant backoff window size, i.e., $8 N$ and $\left(2+\left\lfloor 6 /\left(R^{1 / 2}\right)\right\rfloor\right) N$ in [13] and [14], respectively, where $N$ is the estimated number of stations in the network and $R$ is the channel data rate in Mbps, and the scheme is called Enhanced Collision Avoidance (ECA).

As indicated in the most of studies [2], [3], [4], [5], [6], [7], [8], [9], when the number of competing stations increases, the performance dramatically decreases. In this paper, we propose a novel scheme for contention-based MAC called Backoff Counter Reservation and Classifying Stations (BCRCS) to reduce collisions and improve the system performance. The main reason of causing collisions in the DCF is that stations do not know other stations' information such as backoff counters, specifically two stations with the same backoff counter will definitely collide each other sometime later. If backoff counters of various stations can be known in advance, unnecessary collisions and wasted waiting time can be avoided. Therefore, in the BCR-CS scheme, backoff counters of next frames, if available, are generated in advance and announced in frame transmissions. We further classify stations into three groups: idle, reserved, and contentious groups. Stations in the idle group have no frame ready to transmit. Stations in the reserved group have frames ready to transmit and frames' backoff counters have been successfully announced through the previous successfully transmitted frames so that other stations know the information. Stations in the contentious group have frames ready to transmit, but these frames' backoff counters have not been successfully announced to other stations yet. Frames from stations in the reserved group do not collide each other if there are no hidden nodes since their next frames' backoff counters are known by other stations to avoid using of "colliding" backoff counters. However, collisions may happen among stations in the contentious group since their next frames' backoff counters have not been known by other stations yet. We propose two backoff schemes for the BCR-CS scheme based on the estimated number of stations in the contentious group. The two schemes are denoted as the BCR-CS-b scheme and the BCR-CS-p scheme. They differ only with the backoff schemes: the BCR-CS-b scheme adopts the original binary exponential backoff, but with different chosen parameters, and the BCRCS-p adopts an optimal pseudo-p-persistent scheme discussed later. We also propose two methods to estimate the number of stations in the contentious group/state, denoted as $N_{C}$. We compare the two proposed BCR-CS schemes with the ECA scheme [13], [14] and the original DCF scheme with extensive simulations in which inaccurate estimation and the hidden node problem are also taken into consideration. Note that the proposed schemes are intended to reduce collisions due to the contention-based nature, but not for solving the hidden node problem which are handled by the request-tosend (RTS) and clear-to-send (CTS) mechanism [1].
The rest of the paper is organized as follows. Section 2 describes the proposed BCR-CS schemes in detail. We propose two backoff schemes in Section 3. Section 4 proposes two methods to estimate $N_{C}$, which is related to the backoff schemes. Optimality study for the pseudo-p-persistent scheme is presented in Section 5 in both analytical results and simulation results. In Section 6, performance studies and comparisons are conducted via extensive simulations under two classes of traffic. In Section 7, performance studies and comparisons are conducted via extensive simulations under a new metric, $q$, which is the probability that an outgoing frame arrives when the queue is not empty in a station. We conclude this paper in Section 8.

\section{Backoff Counter Reservation and Classifying Stations}

In this section, we propose a novel scheme for the contention-based MAC called Backoff Counter Reservation and Classifying Stations (BCR-CS). Note that the BCR-CS scheme is later classified into the BCR-CS-b scheme and the BCR-CS-p scheme, based on two different backoff schemes.

In the BCR-CS scheme, the backoff counter of the next frame, if available, is generated in advance and announced in the header of the current frame's transmission. In other words, before a station transmits the current frame, if it has the next frame waiting in the queue, it generates a random backoff counter for the next frame and embedded it in the current frame's MAC header to be announced to other stations. If the current frame is successfully transmitted, other stations know the next backoff counter of the station. Otherwise, other stations have no way to know the station's next backoff counter.

Any station denoted as $S$ has to maintain other stations' backoff counters locally in a table, called Backoff Counter Table (BCT), shown in Fig. 1a. In this example of the BCT, S's own backoff counter is 4 and those of stations $A, B$, and D are 6,2 , and 9 , respectively. Station $C$ has already transmitted its previous frame which does not include its next frame backoff information and -1 is thus used to denote the case when a station's frame's backoff counter is not available. If $S$ does not have a frame to transmit itself, $S$ must also maintain the BCT with its backoff counter as -1 . Whenever $S$ hears an announcement of the next frame's backoff counter of another station F, S updates F's backoff counter in S's local BCT. Whenever S's backoff counter is decreased due to channel access rule such as detecting channel idle, other stations' backoff counters in S's BCT are also decreased unless the counters are -1 . Whenever $S^{\prime}$ s backoff counter freezes due to a busy channel, other stations' backoff counters in S's local table also freeze. If $S$ does not have a backoff counter, after detecting channel idle (busy), other stations' backoff counters in S's BCT are also decreased (suspended) unless the counters are -1 , based on channel access rule. Note that interframe spaces, such as DIFS, SIFS, etc., are included as busy periods. Before S's transmitting a frame, referred to as the current frame, if $S$ has another frame, referred to as the next frame, in the waiting queue, a random backoff is generated for the next frame and embedded in the current frame's MAC header to announce to other stations. If $S$ is in the contentious state, whenever it receives a frame of the station $\mathrm{F}$ indicating that $\mathrm{F}$ has chosen the same backoff counter, $S$ needs to choose another backoff counter since a collision will certainly occur.

Furthermore, as shown in Fig. 1b, a typical station $S$ has three states: idle, reserved, and contentious. $S$ is in the idle 


\begin{tabular}{|l|c|c|c|c|c|c|}
\hline Stations & Self & A & B & C & D & . . . \\
\hline Counters & 4 & 6 & 2 & -1 & 9 & $\ldots$ \\
\hline
\end{tabular}

(a)

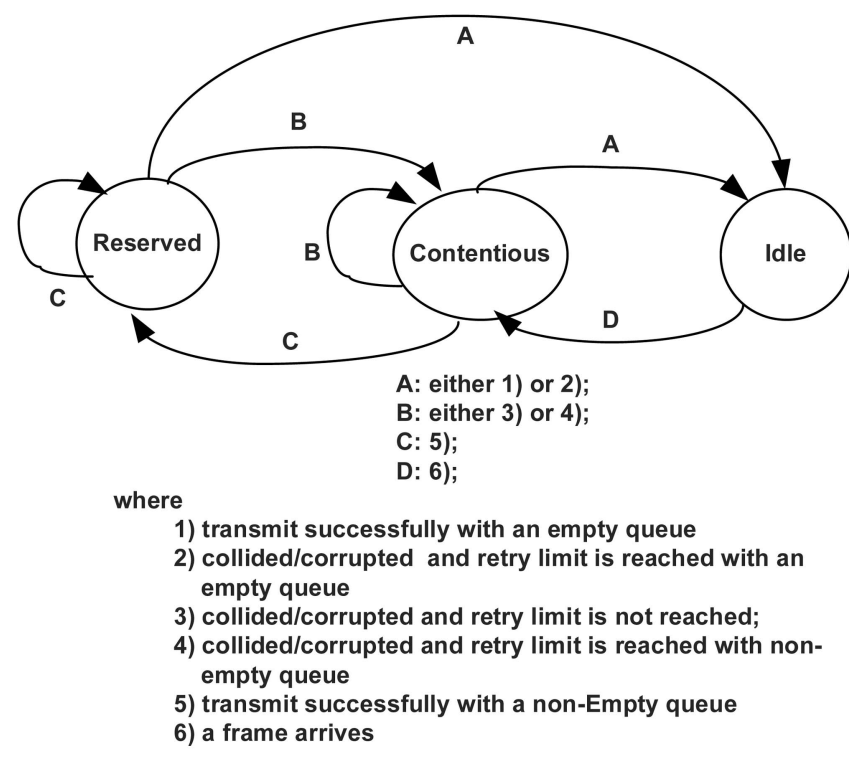

(b)

Fig. 1. (a) Backoff Counter Table (BCT) and (b) station S's state diagram.

state if it has no frame ready to transmit. $S$ is in the reserved state if it has a frame ready to transmit and this frame's backoff counter has been successfully announced through the previous successfully transmitted frame so that other stations know this information. $\mathrm{S}$ is in the contentious state if it has a frame ready to transmit, but this frame's backoff counter has not been successfully announced to other stations. All the stations in the idle state, the reserved state, and the contentious state form an idle group, a reserved group, and a contentious group, respectively. We explain $\mathrm{S}^{\prime}$ s state diagram in Fig. $1 \mathrm{~b}$ as follows:

1. $S$, in the idle state, changes to the contentious state when a frame arrives (Condition D).

2. $\mathrm{S}$, in the contentious state, changes to the reserved state if a frame, denoted as the current frame, is transmitted successfully and there is another frame, denoted as the next frame, in the queue so that the next frame's backoff is generated in advance and announced through the current frame's transmission.

3. $\mathrm{S}$, in the contentious state, remains in the contentious state if the current frame's transmission is collided/ corrupted and the retry limit is not reached.

4. $\mathrm{S}$, in the contentious state, remains in the contentious state if the current frame's transmission is collided/ corrupted, the retry limit is reached, and there is another frame in the queue.

5. S, in the contentious state, changes to the idle state if the current frame's transmission is collided/corrupted, the retry limit is reached, and there is not another frame in the queue.
6. S, in the contentious state, changes to theidle state if the current frame's transmission is transmitted successfully and there is not another frame in the queue.

7. $S$, in the reserved state, remains in the reserved state if a frame is transmitted successfully and there is another frame in the queue.

8. $\mathrm{S}$, in the reserved state, changes to the contentious state if the current frame's transmission is collided/ corrupted and the retry limit is not reached.

9. $\mathrm{S}$, in the reserved state, changes to the contentious state if the current frame's transmission is collided/ corrupted, the retry limit is reached, and there is another frame in the queue.

10. $S$, in the reserved state, changes to the idle state if the current frame's transmission is collided/corrupted, the retry limit is reached, and there is not another frame in the queue.

11. $S$, in the reserved state, changes to the idle state if the current frame's transmission is transmitted successfully and there is not another frame in the queue.

Stations in the reserved group do not have collisions if there are no hidden nodes since their next frames' backoff counters are known by other stations which avoid using conflicted backoff counters. Collisions may happen among stations in the contentious group since their next frames' backoff counters have not been known by other stations yet. If hidden nodes exist, stations in the reserved state/group, called reserved stations, may get collisions and in this case, the station will change from the reserved state into the contentious state, as shown in Fig. 1a.

\section{BACKOFF SCHEMES}

The next frame's backoff counter is chosen uniformly and randomly from the set $\left\{a_{1}, a_{2} \ldots, a_{C W}\right\}$, where $C W$ is the backoff window size and $a_{1}<a_{2}<\ldots<a_{C W}$. For any integer $m$, where $a_{C W} \geq m \geq 0$, we have either $m \in$ $\left\{a_{1}, a_{2} \ldots, a_{C W}\right\}$ or $m$ equals to one of other stations' backoff counters in the station's BCT table. In other words, the set includes the next $C W$ available/unreserved slots. The purpose of the above choices is to avoid choosing conflicted slots already reserved by other stations.

Let $N_{R}, N_{C}$, and $N_{I}$ denote the numbers of stations in the reserved, contentious, and idle groups, respectively. Backoff schemes are proposed in the following subsections.

\subsection{BCR-CS-p}

Based on the estimated value of $N_{C}$, discussed in the next section, we propose the BCR-CS with pseudo-p-persistent backoff (BCR-CS-p) scheme. In this scheme:

1. Any station in the contentious state chooses the most recently available and smallest backoff counter $a_{1}$, i.e., $C W=1$.

2. When the backoff counter reaches zero, it transmits the frame with probability $p$.

3. If the frame is not transmitted, repeat the Steps 1 and 2.

The above scheme seems to be a $p$-persistent backoff scheme, but it is not since in a $p$-persistent scheme, there is no backoff counter. In fact, the above scheme is still CSMA/CA with a minor difference, i.e., any station's behaviors in the contentious state differs from the original CSMA/CA by the way of choosing the backoff counter and the way of 
transmitting with probability $p$. But, it still freezes the backoff counter when seeing a busy channel and decrements the back off counter when seeing an idle channel after a DIFS time. The above features are not those of a $p$-persistent scheme. Furthermore, stations in the reserved state still follow the original CSMA/CA. Therefore, we call this backoff scheme as pseudo-p-persistent backoff, which is only applied to stations in the contentious state. For the proposed BCR-CS-p scheme, $C W$ values of stations in the reserved state are doubled if collided (this case happens only if there are hidden nodes), whereas $C W$ values of stations in the contentious state remain fixed, i.e., $C W=1$, if collided. The initial window size is chosen as $C W=N_{R}+N_{C}$ for the reserved stations. We will study how to choose optimal $p$ value for the contentious group in a later section.

\subsection{BCR-CS-b}

In the proposed BCR-CS with binary exponential scheme, called BCR-CS-b, the original binary exponential backoff is still used. However, the initial window size is chosen as $N_{R}+N_{C}$ (the number of reserved stations + the number of contentious stations). If a collision happens, the window size is doubled until the maximum window size is reached.

The initial window size value of the stations in the reserved state can use the default DCF value. However, since the stations in the reserved group never collide under the condition that there are no hidden nodes, the initial window size value should choose a small value. Note that the probability of the hidden node, if exists, is very small in a WLAN. If initial window size $=1$ for the stations in the reserved state, the throughput will be maximized, however, this may cause starvation for stations in the contentious state. We have $N_{R}+N_{C}$ as the initial window size.

\section{Estimation of $N_{C}$}

In this section, we propose two approaches to estimate $N_{C}$ value, and describe them in Sections 4.1 and 4.2. We can easily estimate $N_{R}$, which is the total number of reserved stations, whose counters are larger than zero in the BCT (Fig. 1a), i.e., $N_{R}$ is the number of positive backoff counters in the BCT. The critical question is how to estimate $N_{C}$, the number of stations in the contentious group. Counting " -1 " in the BCT may lead us an approximation of $N_{C}+N_{I}$, but not necessary $N_{C}$ since, for example, some stations may move out of the radio range, turn out computers, or turn out the WLAN cards. There is no easy way to obtain the accurate $N_{C}$ instant value no mater what method we use. For example, assuming that there are two new frame arrivals for an idle station, the system has no way to predict arrivals of these new frames for the idle station. However, the system can learn by mistakes and learn when the system is stable. Therefore, the $N_{C}$ value must be approximated on an average value. $N_{C}$ can be obtained via measurements over previous intervals. Since the $N_{C}$ value is obtained in the previous measurement interval, it might not equal to the actual $N_{C}$ value in the current system. $N_{C}$ is essentially an average measured value over all stations, while the actual $N_{C}$ value is an instant value of the system. The length of measurement interval and the traffic pattern will affect how accurate the measured $N_{C}$. Therefore, we need to show that influence of a nonaccurate $N_{C}$ value on performance results should not be large. We propose two approaches in following sections.

\subsection{Estimated-by-Time}

We propose the first approach, called Estimated-by-Time (EBT), to estimate the value of $N_{C}$. Each station measures its activities during three states as follows. Let $T_{R}(j), T_{C}(j)$, and $T_{I}(j)$ denote the portions of time spending in the reserved state, the contentious state, and the idle state, respectively, for the station $j$. We have: $T_{R}(j)+T_{C}(j)+T_{I}(j)=1$. Whenever station $j$ transmits a frame, it piggybacks the $T_{R}(j)$ and $T_{C}(j)$ values. The $T_{I}(j)$ value can be derived based on the $T_{R}(j)$ and $T_{C}(j)$ values. Therefore, other stations also know them $\left(T_{R}(j), T_{C}(j)\right.$, and $\left.T_{I}(j)\right)$ and periodically update their values for station $j$. Let $M\left(\Delta_{t}, t\right)$ denote the number of piggybacked frames from different stations obtained during the time period from $t-\Delta_{t}$ to $t$, where $\Delta_{t}$ is a relative large value. Multiple frames from the same station count only once, i.e., the most current one. Let $V$ stand for the number of " -1 " in the BCT table shown in Fig. 1a. We have

$$
V \approx N_{C}+N_{I}
$$

To make (1) more accurate, each station deletes stations that have" -1 " in the BCT for a time period longer than a large predefined threshold. In other words, those stations that are the idle state for a very long time (mostly because of moving away) are deleted from the BCT. Then, current measured $N_{C}(t)$ value can be approximated by

$$
N_{C, \text { current }}(t)=\sum_{j=1}^{M(\Delta t, t)} T_{C}(j) .
$$

Note that some idle stations may not necessarily send the piggyback frames, but this information does not have impacts on estimation of the value $N_{C}$. Let $t^{\prime}$ denote the previous measurement time, and $\alpha(0 \leq \alpha \leq 1)$ denote a weight value. An exponential smoothing technique can be used to obtain estimated $N_{C}(t)$ value as follows:

$$
N_{C}(t)=\min \left(\alpha N_{C}\left(t^{\prime}\right)+(1-\alpha) N_{C, \text { current }}(t), V\right) .
$$

To piggyback $T_{R}(j)$ and $T_{C}(j)$ values, additional fields are needed in the MAC header format, i.e., put additional two fields at the end of the MAC header to carry the information. Since the proposed mechanisms change the behaviors and protocols of the MAC layer, it is reasonable to have a modified MAC header format, while the additional fields are added at the end of the MAC header to minimize the incompatibility with the original MAC format.

\subsection{Estimated-by-Probability}

In this section, we propose another approach, called Estimated-by-Probability (EBP), to estimate the value of $N_{C}$. Each station measures its activities as follows. Let $q(j)$ denote the ratio of the number of reserved frames and the total number of frames during a measured interval, for the station $j$, where a reserved frame is a frame in the reserved state. Then, $q(j)$ can approximately be the probability that a transmitted frame carries the next frame's backoff counter, i.e., the probability that the next frame already arrives before the current frame is transmitted. Each station piggybacks its $q(j)$ value into its frames. Whenever station $j$ transmits a frame, it piggybacks the $q(j)$ value. Therefore, other stations also know their $q(j)$ and periodically update 
the value for station $j$. Then, current measured $N_{C}(t)$ value can be approximated by

$$
N_{C, \text { current }}(t)=\sum_{j=1}^{M(\Delta t, t)} q(j) .
$$

Note that some idle stations may not necessarily send the piggyback frames, but this information does not have impacts on estimation of the value $N_{C}$. Equation (3) can also be used to smooth the estimated value. To piggyback backoff counter, $q(j)$ value, an additional field is needed in the MAC header format, i.e., an additional field at the end of the MAC header to carry the information.

\section{Optimality Study for the P-Persistent BACKOFF}

In this section, we provide an optimality study for the $p$-persistent backoff scheme. Note that the $p$-persistent backoff scheme and the pseudo- $p$ persistent backoff scheme are different as follows. The pseudo-p persistent backoff scheme is the $p$-persistent backoff scheme when applied to the BCR-CS scheme as described before. In Section 5.1, we provide an optimal analysis for the $p$-persistent backoff. In Section 5.2, we evaluate the $p$-persistent backoff scheme with different parameter choices.

\subsection{Optimality Analysis}

The question is how to obtain the optimal $p$ value. Assume that $M$ stations are competing the channel at a timeslot. Let $p_{s u c}, p_{c o l}$, and $p_{i d l}$ denote the probabilities of a successful transmission, a collision, and an idle slot, respectively; let $T_{s u c}, T_{c o l}$, and $T_{i d l}$ denote the corresponding times of a successfully transmission, a collision, and an idle slot, respectively. Let $\beta$ denote $T_{i d l} / T_{c o l}$. The normalized utilization is given as follows:

$$
\begin{aligned}
& U(p)=\frac{p_{\text {suc }} T_{\text {suc }}}{p_{\text {suc }} T_{\text {suc }}+p_{\text {col }} T_{\text {col }}+p_{\text {idl }} T_{\text {idl }}} \\
& =\frac{1}{1+\frac{T_{\text {col }}}{T_{\text {suc }}}\left(\frac{p_{\text {col }}}{p_{\text {suc }}}+\beta \frac{p_{\text {idl }}}{p_{\text {suc }}}\right)},
\end{aligned}
$$

where we have

$$
\begin{aligned}
& p_{\text {suc }}=M p(1-p)^{M-1}, \\
& p_{\text {idl }}=(1-p)^{M}, \\
& p_{\text {col }}=1-p_{\text {suc }}-p_{\text {idl }} .
\end{aligned}
$$

If $M=1$, it's obvious that utilization is maximized when $p=1$.

If $M>1$, since when $p=0$ or $p=1, U(p)=0$, we only consider $p \in(0,1)$. To maximize $U(p)$ is to minimize $f(p)$, which is defined as follows:

$$
\begin{aligned}
& f(p)=\frac{p_{\text {col }}}{p_{\text {suc }}}+\beta \frac{p_{\text {idl }}}{p_{\text {suc }}} \\
& =M^{-1} p^{-1}(1-p)^{1-M}+M^{-1}(\beta-1) p^{-1}(1-p)-1
\end{aligned}
$$

where $\beta \in(0,1)$.

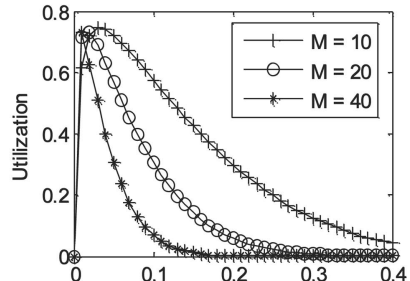

(a) $p$

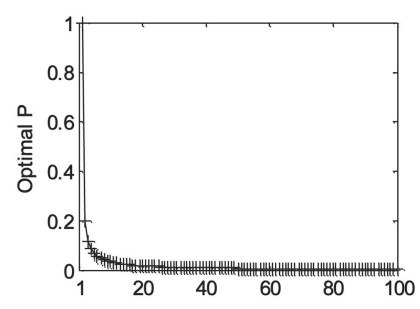

(b) M

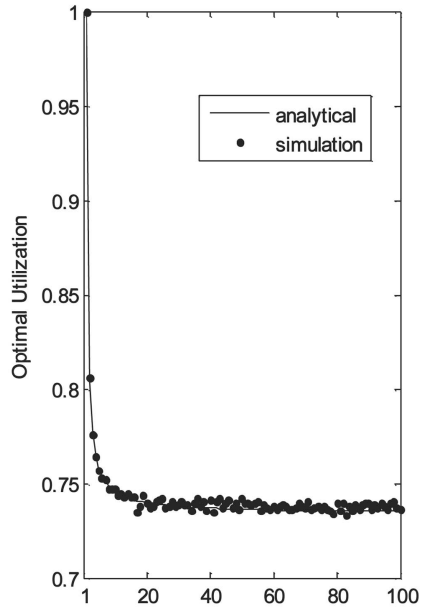

(c) $\mathrm{M}$

Fig. 2. (a) Utilization, (b) the optimal p value, and (c) optimal utilization validated by simulations.

$f(p)$ is a continuous and infinitely differentiable function when $p \in(0,1)$. To obtain the minimal value of $f(p)$, we obtain its first derivative as follows:

$$
\frac{\partial f(p)}{\partial p}=-M^{-1} p^{-2}\left[(1-M p)(1-p)^{-M}+\beta-1\right] .
$$

When $\partial f(p) / \partial p=0$, we have

$$
(1-M p)(1-p)^{-M}+\beta-1=0 .
$$

Equation (11) can be easily solved numerically.

\subsection{Evaluation}

In this section, we provide an evaluation on the $p$-persistent scheme. We shows the optimal $\mathrm{p}$ value, the optimal utilization, and the effects of estimated $M$ values on the utilization in the following sections. Furthermore, we conduct simulations to validate the analytical results. The simulation program is conducted with $\mathrm{C}++$ using discrete event simulation. Note that this section is to evaluate the $p$-persistent backoff only when the BCR-CS is not considered. In experiments, we adopt the following parameters: $T_{\text {suc }}=153 \mu \mathrm{s}, T_{\text {col }}=153 \mu \mathrm{s}$, and $T_{\text {idl }}=9 \mu \mathrm{s}$. Moreover, each station always has at least one frame ready to transmit.

\subsubsection{Optimal $p$ Value}

Fig. 2a shows the normalized utilization over the $p$ value for different $M$ values. We observe that there exists an optimal $p$ value given the $M$ value, which matches the solution from (9). Furthermore, as $M$ increases, the optimal $p$ value decreases. Fig. $2 \mathrm{~b}$ shows the optimal $p$ value over $M$, and the optimal $p$ value decreases as $M$ increases.

\subsubsection{Optimal Utilization Validated with Simulations}

Fig. 2c shows the optimal utilization versus $M$, the number of stations. As the $M$ value increases, the optimal utilization decreases until reaching about 73 percent, and then stays flat. The figure also shows that the analytical results match simulation results very well. 


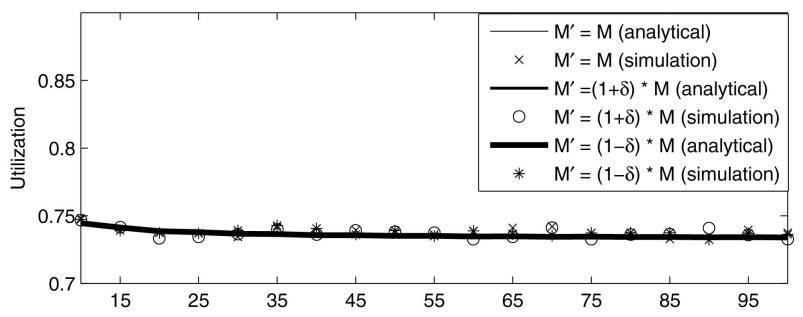

(a)

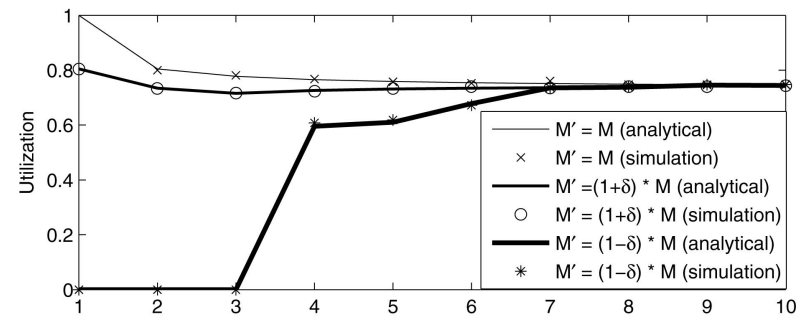

(b)

Fig. 3. Effects of estimated $M$ values. (a) $\mathrm{M}$ and (b) $\mathrm{M}$.

\subsubsection{Effects of Estimated $M$ Values Validated with Simulations}

This section studies the effects of estimated $M$ values on utilization under the assumption that the estimated $M$ values may be inaccurate by $\pm \delta$. In this experiment, we choose $\delta=$ $10 \%$ when $M \geq 10$, but we believe that when $M$ is small, the relative error may be larger, and then we choose $\delta$ differently when $M$ is small. We have

$$
\delta=\left\{\begin{array}{l}
10 \%, M \geq 10 \\
1-(M-1) \times 10 \%, 1 \leq M<10 .
\end{array}\right.
$$

In other words, when $M=1, \delta=100 \%$; when $M=2$, $\delta=90 \% ; \ldots$; and when $M \geq 10, \delta=10 \%$. Fig. 3a shows the effect of measurement error of $M$ to utilization when $100 \geq M \geq 10$. The results show that overestimated $M$ and underestimated $M$ do not affect utilization much. Fig. 3b shows the effect of measurement error of $M$ to utilization when $10 \geq M \geq 1$. Since we choose a larger $\delta$ when $M$ is smaller than 10, we see a big impact on utilization when $M$ is very smaller $(<4)$ with underestimated utilization $(M-\delta)$, i.e., utilization is deteriorated dramatically. The reason is that for example when $M=1$, the underestimated $M$, denoted as $M^{\prime}$, is $M-\delta=0$ so that it is assumed that there is no another station to transmit, but in fact there is another station which will compete the channel. Therefore, in our proposed scheme, when the estimated $M^{\prime}$ is smaller than 5, we always adjust $M^{\prime}$ to 5 . Moreover, from Fig. $3 \mathrm{~b}$, it is obvious that when $M$ is very small, overestimation is always better than underestimation, although utilization is slightly worse than that with the accurate value. Fig. 3 also shows that analytical results match very well with simulation results.

\section{Performance Evaluation under Two Classes of Traffic}

In this section, we provide a comprehensive evaluation for the proposed schemes as well as a comparison with the DCF and the ECA [13], [14]. All results in this section are simulation results, which are obtained via simulation programs coded by JAVA language using discrete event simulation. For the DCF scheme, the IEEE 802.11a is chosen with the following parameters: frame payload is 500 bytes; beacon interval is $100 \mathrm{~ms}$; DIFS time is $34 \mu \mathrm{s}$; SIFS time is $16 \mu \mathrm{s}$; slot time is $9 \mu \mathrm{s}$; physical preamble is $16 \mu \mathrm{s}$; physical header time is $4 \mu \mathrm{s}$; symbol time is $4 \mu \mathrm{s}$; the control rate is $24 \mathrm{Mbps}$; the data rate is $54 \mathrm{Mbps}$; the backoff minimal window size is 32 ; and the maximum backoff window size is 1,024. For the proposed schemes and the ECA, most of the above parameters are also the same.

In the simulations, we define two types of stations: type A and type B, where a type A station always has at least a frame ready to send in the queue at any time, and in a type B station, a frame only arrives after the previous frame is just transmitted. Therefore, a type A station can fully utilize the reservation benefits, i.e., broadcasting next backoff counter in the current fame, whereas a type B station can not use the reservation benefits at all. Therefore, the reservation scheme has no effect on type $B$ stations. Let $N_{A}$ and $N_{B}$ denote the numbers of the type A stations and the type $B$ stations, respectively. In simulations, the number of stations is always $100=N_{A}+N_{B}$. A traffic pattern is denoted as $N_{A} / N_{B}(0 / 100,20 / 80,40 / 60,50 / 50, \ldots, 100 / 0)$. For example, 20/80 means that there are 20 type A stations and 80 type B stations.

\subsection{Pseudo-p-Persistent}

In Section 5.2, we evaluate the $p$-persistent backoff scheme via both simulation results and analytical results, but we do not put it into the BCR-CS scheme. When the $p$-persistent backoff is put into the BCR-CS, it becomes the pseudo-ppersistent backoff explained before since it is not the exact $p$-persistent anymore.

In this section, we evaluate the BCR-CS with the pseudo-ppersistent backoff. We compare the three different $p$ values: the optimal one, $p=1 / N_{C}$, and $p=1 /\left(2 N_{C}\right)$, where $N_{C}$ is the estimated number of contentious stations.

Fig. 4a shows throughput over the traffic pattern $N_{A} / N_{B}(0 / 100,20 / 80,40 / 60,50 / 50, \ldots, 100 / 0)$. As illustrated in the figure, as the number of type A stations increases from 0 to 100, the throughput increases since more stations can take advantage of broadcasting the backoff counter in advance. When $N_{A}=0$ and $N_{B}=100$, reservation becomes useless, whereas when $N_{A}=100$ and $N_{B}=0$, reservation is fully utilized and, therefore, a better throughput is achieved. The figure also shows that the optimal $\mathrm{p}$ has the best throughput among the three choices.

Fig. $4 \mathrm{~b}$ shows the number of collisions over the traffic pattern $N_{A} / N_{B}(0 / 100,20 / 80,40 / 60,50 / 50, \ldots, 100 / 0)$. As illustrated in the figure, as the number of type A stations increases from 0 to 100 , the number of collisions decreases to zero since more stations can take advantage of broadcasting the backoff counter in advance. When $N_{A}=0$ and $N_{B}=100$, reservation becomes useless, whereas when $N_{A}=100$ and $N_{B}=0$, reservation is fully utilized, and therefore, the number of collisions is zero. The figure also shows that the optimal $\mathrm{p}$ has the least number of collisions among the three choices.

\subsection{Comparison over Simulation Time}

In this section, we compare the DCF, the ECA, the proposed BCR-CS, and the BCR-CS with pseudo-p-persistent schemes over simulation time. In this subsection, the number of type A stations is 40 and the number of type B stations is 60 . Initially, there are five stations of type A in the system. 




(a)

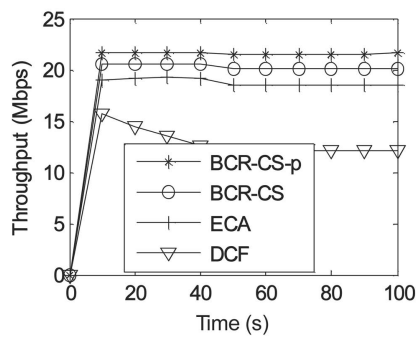

(c)



(b)



(d)

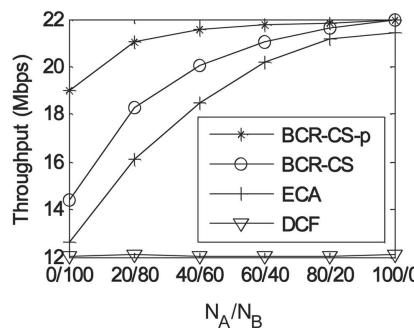

(a)

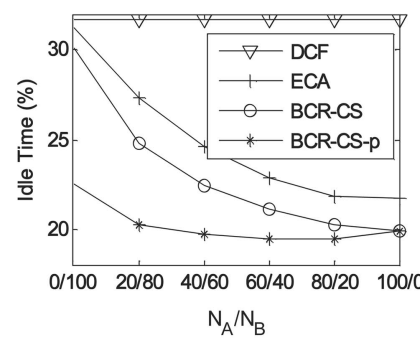

(c)

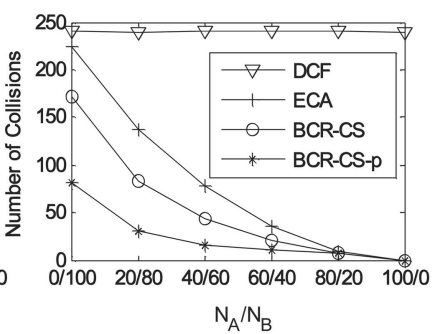

(b)

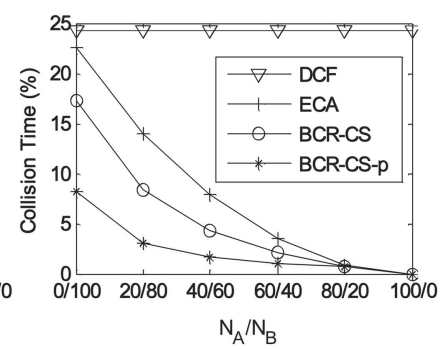

(d)

Fig. 4. (a) Throughput with different $p$ values, (b) number of collisions with different $p$ values, (c) throughput versus simulation time, and (d) number of collisions over simulation time.

Then, five stations join every five seconds alternatively with type B and type A until there are total 100 stations in the system. The total simulation time is 200 seconds.

Fig. 4c and Fig. 4d show throughput and number of collisions, respectively, over the simulation time. As illustrated in the figures, all three schemes are better than the DCF, the BCR-CS is better than the ECA, and the BCRCS with pseudo-p-persistent is the best scheme among the four schemes.

\subsection{Comparison over Traffic Pattern}

In this section, we compare the DCF, the ECA, the proposed BCR-CS, and the BCR-CS with pseudo-p-persistent schemes over traffic pattern. Unlike the previous section, we choose a particular traffic pattern, in this section, we change different traffic patterns.

Fig. 5a shows throughput over the traffic pattern $N_{A} / N_{B}(0 / 100,20 / 80,40 / 60,50 / 50, \ldots, 100 / 0)$. As illustrated in the figure, as the number of type $\mathrm{A}$ stations increases from 0 to 100, throughputs of the ECA, the BCR-CS, and the BCR-CS with pseudo-p-persistent schemes all increase since more stations can take advantage of broadcasting the backoff counter in advance. When $N_{A}=0$ and $N_{B}=100$, reservation becomes useless, whereas when $N_{A}=100$ and $N_{B}=0$, reservation is fully utilized and, therefore, better throughputs are achieved for these three schemes. Furthermore, all three schemes are better than the DCF, the BCR-CS is better than the ECA, and the BCR-CS-p scheme is the best scheme among the four schemes. The DCF has the same throughput at all traffic situations since type A and type B stations make no difference to the DCF.

Fig. $5 \mathrm{~b}$ shows the number of collisions over the traffic pattern $N_{A} / N_{B}(0 / 100,20 / 80,40 / 60,50 / 50, \ldots, 100 / 0)$. As illustrated in the figure, as the number of type A stations increases from 0 to 100 , the numbers of collisions of the ECA, the BCR-CS, and the BCR-CS-p schemes all decrease

Fig. 5. (a) Total throughput, (b) number of collisions, (c) idle time, and (d) collision time.

to zero since more stations can take advantage of broadcasting the backoff counter in advance. When $N_{A}=0$ and $N_{B}=100$, reservation becomes useless for these three schemes, whereas when $N_{A}=100$ and $N_{B}=0$, reservation is fully utilized and, therefore, the numbers of collisions are zero. Furthermore, all three schemes are better than the DCF, the BCR-CS is better than the ECA, and the BCR-CS-p scheme is the best scheme among the four schemes. The DCF has the same number of collisions at all traffic situations since type A and type B stations make no difference to the DCF.

Fig. 5c and Fig. 5d shows idle time and collision time in percentage over the traffic pattern $N_{A} / N_{B}(0 / 100,20 / 80$, $40 / 60,50 / 50, \ldots, 100 / 0)$. As illustrated in the figures, as the number of type A stations increases from 0 to 100 , the idle times and the collision times in percentage of the ECA, the BCR-CS, and the BCR-CS-p schemes all decrease since more stations can take advantage of broadcasting the backoff counter in advance. In fact, the collision times of above three schemes all decrease to zero. When $N_{A}=0$ and $N_{B}=100$, reservation becomes useless for these three schemes, whereas when $N_{A}=100$ and $N_{B}=0$, reservation is fully utilized, and therefore, the collision times are zero. Furthermore, all three schemes are better than the DCF, the BCR-CS is better than the ECA, and the BCR-CS-p scheme is the best scheme among the four schemes. The DCF has the same idle time and collision at all traffic situations since type $A$ and type $B$ stations make no difference to the DCF. From Fig. 5c and Fig. 5d, we can also conclude that the reason that the proposed schemes are better than the ECA is that they reduce more idle time and collision time!

\section{Performance Evaluation under $q$}

In this section, we provide a comprehensive evaluation under the new metric $q$, which is defined as the probability that an 


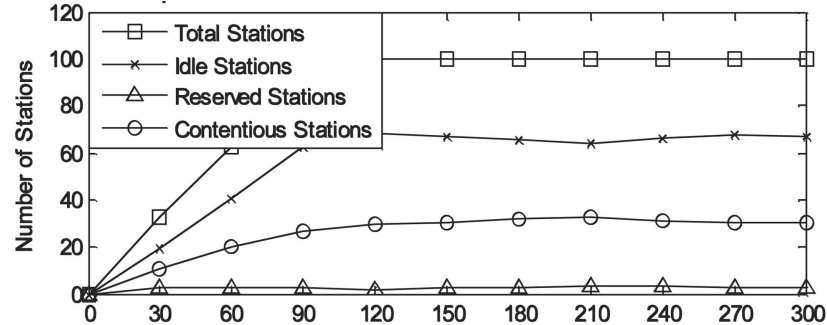

(a) Time (s)



Fig. 6. Number of stations: (a) $E(q)=0$ and (b) $E(q)=1$.

outgoing frame arrives when the queue is not empty in a station. For example, $q=0.3$ means that there is 30 percent chance that the next frame will arrive when the queue is not empty, and 70 percent chance that the next frame will arrive seeing an empty waiting queue. By setting $q$ value of each station, we study the performance of the proposed schemes on different traffic situations. We study performance over the mean, denoted by $E(q)$, and the variance, denoted by $\operatorname{Var}(q)$, of $q$. Two extreme cases of $q$ are stated as follows: If $q$ is always zero, it is equivalent to the class B defined in the previous section. On the other hand, if $q$ is always one, it is equivalent to the class A defined in the previous section. In the other cases, $q$ changes between 0 and 1 . Therefore, the traffic pattern is more general than the traffic pattern that presented in the previous section.

All results in this section are simulation results, which are obtained via simulation programs coded by JAVA language using discrete event simulation. Other parameters are the same as the previous section.

Stations are added in the system as follows: Initially, there are five stations, and then five stations are added after each five seconds until there are 100 stations in the system. The total simulation time is 300 seconds. In other words, in the last 200 seconds, there is no station joining/leaving the system.

Fig. $6 a$ and Fig. $6 b$ shows the number of total stations, the number of idle stations, the number of reserved stations, and the number of contentious stations when $E(q)=0$ and when $E(q)=1$, respectively. But, Fig. 6 does not show the cases when $0<E(q)<1$.

\subsection{Comparison of Schemes under $q$}

Fig. 7a shows throughput over $E(q)$, under different schemes, where $E(q)$ is the mean of $q$. As illustrated in the figure, the BCR-CS-p, the BCR-CS-b, and the ECA all outperform the DCF, and the BCR-CS-p is the best scheme. Furthermore, throughputs of the BCR-CS-p, the BCR-CS-b, and the ECA increase as $E(q)$ increases since as $E(q)$ increases, the probability that a frame can be reserved is larger.

Fig. $7 \mathrm{~b}$ shows collision time (percent) over $E(q)$, under different schemes. As illustrated in the figure, the BCR-CS-p,

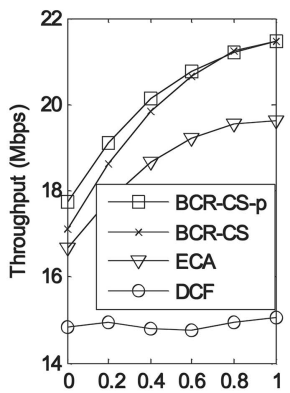

(a) $E(q)$

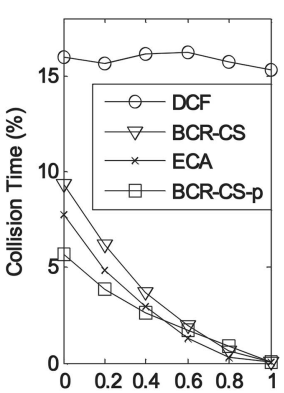

(b) $E(q)$

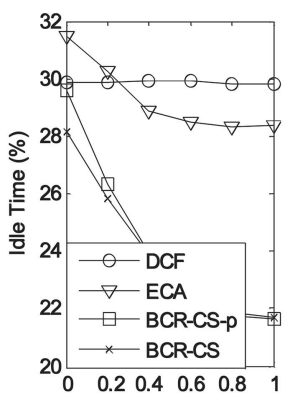

(c) $E(q)$
Fig. 7. (a) Throughput, (b) collision time, and (c) idle time.

the BCR-CS-b, and the ECA all outperform the DCF. The BCRCS-p, the BCR-CS-b, and the ECA are almost similar in terms of collision time. Furthermore, collision times of the BCR-CS$\mathrm{p}$, the BCR-CS-b, and the ECA decrease as $E(q)$ increases since as $E(q)$ increases, the probability that a frame can be reserved is larger.

Fig. 7c shows idle time (percent) over $E(q)$, under different schemes. As illustrated in the figure, the BCR-CS-p and the BCR-CS-b outperform the DCF. The ECA is worse than the DCF when $E(q)$ is small, but is better than the DCF when $E(q)$ is large. Idle times of the BCR-CS-p, the BCR-CS-b, and the ECA decrease as $E(q)$ increases since as $E(q)$ increases, the probability that a frame can be reserved is larger. Comparing Figs. 7a, 7b, and 7c, we observe that the BCR-CS-p and the BCR-CS-b outperform the ECA mostly because the BCR-CS-p and the BCR-CS-b save more idle time than the ECA.

\subsection{Effects of $q$ Distributions}

During our simulations, we have some interesting observations for both the BCR-CS-p and the BCR-CS-b, presented as follows. Figs. $8 \mathrm{a}, 8 \mathrm{~b}$, and $8 \mathrm{c}$ show throughput, collision time, and idle time, respectively, over the variance of $q$, when $E(q)=0.5$. We observe that as the variance of $q$ increases, throughput increases, and collision time and idle time decrease. The collision time of BCR-CS-b is much larger than that BCR-CS-p although the idle time of BCR-CS-b is smaller than that of BCR-CS-p so that eventually the throughput of of BCR-CS-p is a little bit higher than that of BCR-CS-b.

\subsection{Comparison of $N_{C}$ Estimated Methods and Real Value}

This section evaluates throughput, collision time, and idle time under different $N_{C}$ estimation methods: Estimated-byTime (EBT), Estimated-by-Probability (EBP), and real value

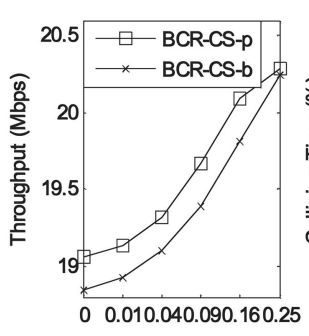

(a) $\operatorname{VAR}(q)$

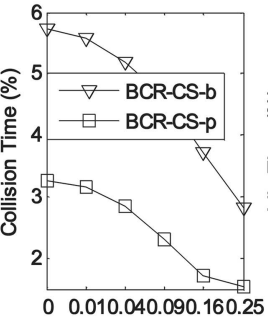

(b) $\operatorname{VAR}(q)$

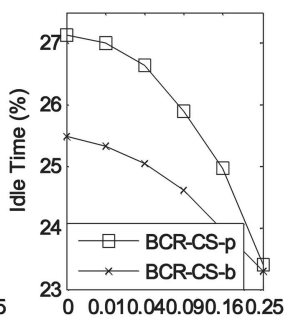

(c) $\operatorname{VAR}(q)$
Fig. 8. (a) Throughput, (b) collision time, and (c) idle time. 


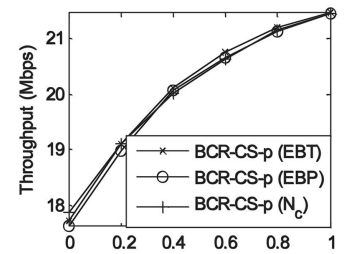

(a) $E(q)$

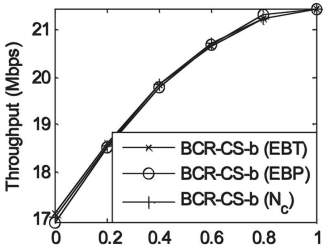

(d) $E(q)$

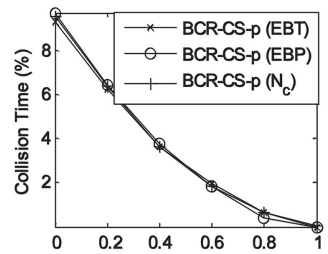

(b) $E(q)$

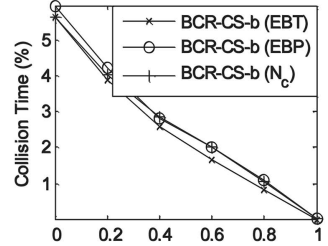

(e) $E(q)$

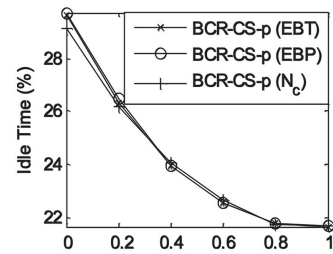

(c) $E$ (q)

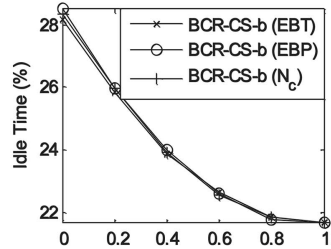

(f) $E(q)$

Fig. 9. (a) and (b) Throughput, (c) and (d) collision time, and (e) and (f) idle time.

$\left(N_{C}\right)$. Figs. 9a, 9b, 9c, 9d, 9e, and 9f show that for both the BCR-CS-p and the BCR-CS-b, any of all three metrics (throughput, collision time, and idle time) is similar under $\mathrm{EBT}, \mathrm{EBP}$, and the real value.

\section{Conclusion}

In this paper, we proposed a novel scheme for contentionbased protocol, called Backoff Counter Reservation and Classifying Stations (BCR-CS), based on the concept of classifying stations as well as announcing the backoff counters in advance. Two different backoff schemes were proposed for the BCR-CS to reduce collisions and improve the system performance. There are three key aspects in the proposed schemes: reservation, classification, and optimality. The proposed scheme outperforms the DCF and ECA because: 1) announcing the backoff counters in advance avoids possible collisions among reserved stations; and 2) classifying stations enable one to estimate the number of contentious stations, thus determining the appropriate window size to reduce collisions among contentious stations. Furthermore, two estimation methods of the number of contentious stations are proposed. Extensive simulation studies were performed to compare with the new protocol with the DCF and ECA, and our results show that the proposed scheme is the best in terms of throughput and delay. The proposed schemes outperform the ECA mostly due to reduction of idle time. An interesting observation is that with a larger variance of $q$ distribution, throughput is larger for the proposed schemes. We also show that the proposed estimation methods perform very well in terms of throughput, collision time, and idle time.

\section{ACKNOWLEDGMENTS}

An earlier and short version of this paper was presented at the Proceedings of the Conference on Networking 2005 [16].

\section{REFERENCES}

[1] IEEE 802.11: Wireless LAN Medium Access Control (MAC) and Physical Layer (PHY) specification, 1999.

[2] F. Calì, M. Conti, and E. Gregori, "Dynamic Tuning of the IEEE 802. 11 Protocol to Achieve a Theoretical Throughput Limit," IEEE/ACM Trans. Networking, vol. 8, no. 6, pp. 785-790, Dec. 2000.
[3] F. Calì, M. Conti, and E. Gregori, “IEEE 802.11 Protocol: Design and Performance Evaluation of an Adaptive Backoff Mechanism," IEEE J. Selected Areas in Comm., vol. 18, no. 19, pp. 1774-1786, Sept. 2000.

[4] G. Bianchi, "Performance Analysis of the IEEE 802. 11 Distributed Coordination Function," IEEE J. Selected Areas in Comm., vol. 18, no. 3, pp. 535-547, Mar. 2000.

[5] E. Ziouva and T. Antonakopoulos, "CSMA/CA Performance under High Traffic Conditions: Throughput and Delay Analysis," Computer Comm., vol. 25, pp. 313-321, 2002.

[6] B. Bing and R. Subramanianb, "A Novel Technique for Quantitative Performance Evaluation of Wireless LANs," Computer Comm., vol. 21, no. 9, pp. 833-838, July 1998.

[7] K.C. Huang and K.-C. Chen, "Interference Analysis of Nonpersistent CSMA with Hidden Terminals in Multicell Wireless Data Networks," Proc. IEEE Int'l Symp. Personal Indoor and Mobile Radio Comm., pp. 907-911, Sept. 1995.

[8] H.S. Chhaya and S. Gupta, "Performance Modeling of Asynchronous Data Transfer Methods of IEEE 802. 11 MAC Protocol," Wireless Networks, vol. 3, pp. 217-234, 1997.

[9] Y.C. Tay and K.C. Chua, "A Capacity Analysis for the IEEE 802.11 MAC Protocol," Wireless Networks, vol. 7, pp. 159-171, 2001.

[10] Y. Xiao and J. Rosdahl, "Throughput and Delay Limits of IEEE 802. 11," IEEE Comm. Letters, vol. 6, no. 8, pp. 355-357, Aug. 2002.

[11] Y. Xiao and J. Rosdahl, "Performance Analysis and Enhancement for the Current and Future IEEE 802. 11 MAC Protocols," ACM SIGMOBILE Mobile Computing and Comm. Rev. (MC2R), vol. 7, no. 2, pp. 6-19, Apr. 2003.

[12] K.K. Leung, B. McNair, L.J. Cimini, and J.H. Winters, "Outdoor IEEE 802. 11 Cellular Networks: MAC Protocol Design and Performance," Proc. IEEE Int'l Conf. Comm. '02, Apr. 2002.

[13] R.O. Baldwin, N.J. Davis IV, and S.E. Midkiff, "A Real-Time Medium Access Control Protocol for Ad Hoc Wireless Local Area Networks," Mobile Computing and Comm. Rev. (MC2R), vol. 3, no. 2, pp. 20-27, Apr. 1999.

[14] R.O. Baldwin, N.J. Davis IV, S.E. Midkif, and R.A. Raines, "Packetized Voice Transmission Using RT-MAC, a Wireless Real-Time Medium Access Control Protocol," Mobile Computing and Comm. Rev. (MC2R), vol. 5, no. 3, pp. 11-25, July 2001.

[15] IEEE, Part 11a: Wireless LAN Medium Access Control (MAC) and Physical Layer (PHY) specifications High-speed Physical Layer in the $5 \mathrm{GHz}$ Band, 1999.

[16] Y. Xiao, H. Li, K. Wu, K.K. Leung, and Q. Ni, "Reservation and Grouping Stations for the IEEE 802.11 DCF," Proc. Conf. Networking, pp. 395-405, 2005.

[17] Y. Xiao and Y. Pan, "Differentiation, QoS guarantee, and Optimization for Real-time Traffic over One-Hop Ad Hoc Networks," IEEE Trans. Parallel and Distributed Systems, vol. 16, no. 6, pp. 538-549, June 2005.

[18] Y. Xiao and H. Li, "Voice and Video Transmissions with Global Data Parameter Control for the IEEE 802.11E Enhance Distributed Channel Access," IEEE Trans. Parallel and Distributed Systems, vol. 15, no. 11, pp. 1041-1053, Nov. 2004. 
[19] Y. Xiao and H. Li, "Local Data Control and Admission Control for Ad Hoc Wireless Networks," IEEE Trans. Vehicular Technology, vol. 53, no. 5, pp. 1558-1572, Sept. 2004.

[20] Y. Xiao, "IEEE 802.11 Performance Enhancement via Concatenation and Piggyback Mechanisms," IEEE Trans. Wireless Comm., vol. 4, no. 5, pp. 2182-2192, Sept. 2005.

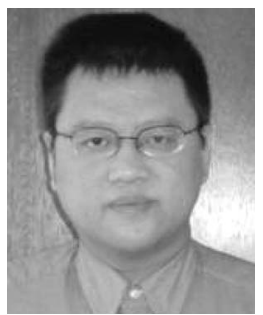

Yang Xiao worked at Micro Linear as an MAC (Medium Access Control) architect involving the IEEE 802.11 standard enhancement work before he joined the Department of Computer Science at The University of Memphis in 2002. Dr. Xiao is a senior member of the IEEE and IEEE Computer Society. He was a voting member of IEEE 802.11 Working Group from 2001 to 2004. He currently serves as Editor-inChief for the International Journal of Security and Networks (IJSN) and for the International Journal of Sensor Networks (IJSNet). He serves as an associate editor or on the editorial boards for the following refereed journals: International Journal of Communication Systems (Wiley), Wireless Communications and Mobile Computing(WCMC) (Wiley), EURASIP Journal on Wireless Communications and Networking (WCN), and International Journal of Wireless and Mobile Computing (IJWMC). He serves as a (lead) journal guest editor for IJSN, special issue on security issues in sensor networks in 2005, as a (lead) journal guest editor for EURASIP WCN, special issue on wireless network security in 2005 , as a (sole) journal guest editor for the Computer Communications Journal (Elsevier), special issue on energy-efficient scheduling and MAC for sensor networks, WPANs, WLANs, and WMANs in 2005, as a (lead) journal guest editor for WCMC (Wiley), special issue on mobility, paging, and quality of service management for future wireless networks in 2004, and as a (lead) journal guest editor for IJWMC, special issue on medium access control for WLANs, WPANs, ad hoc networks, and sensor networks in 2004. He serves as a referee/reviewer for many funding agencies, as well as a panelist for the US National Science Foundation. Dr. Xiao's research areas include wireless networks and network security.

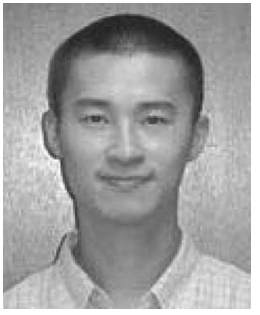

Frank Haizhon Li received the MS and $\mathrm{PhD}$ degrees in computer science from the University of Memphis in 2003 and 2005, respectively. $\mathrm{He}$ is an assistant professor at the University of South Carolina, Upstate. His main research interests are wireless networks and computer security. He also serves as a reviewer for a number of journals and TPC member in a number of conferences. He is a student member of the IEEE.

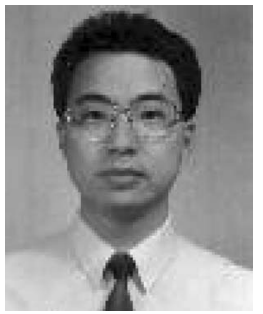

Kui Wu received the $\mathrm{PhD}$ degree in computing science from the University of Alberta, Canada, in 2002. He then joined the Department of Computer Science, University of Victoria, Cana$\mathrm{da}$, where he is currently an assistant professor. His research interests include mobile and wireless networks, sensor networks, network performance evaluation, and network security. He has been actively serving as a TPC member in several international conferences, such as Globecom, ICC, LCN, IPCCC, and ADHOC-NOW, and as a reviewer for several international journals, such as ACM WINET, ACM MONET, IEEE/ACM Transactions on Networking, IEEE Transactions on Parallel and Distributed Systems, IEEE Transactions on Wireless Communications, Wiley WCMC, and Elsevier Computer Networks. He cochaired the first IEEE LCN workshop on network security. He is a member of the IEEE and the IEEE Computer Society.

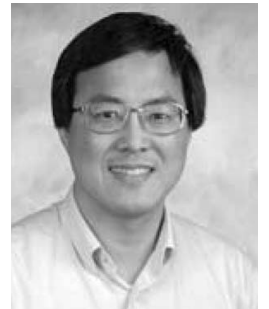

Kin K. Leung received the BS degree from the Chinese University of Hong Kong in 1980, and the $\mathrm{MS}$ and $\mathrm{PhD}$ degrees in computer science from the University of California, Los Angeles, in 1982 and 1985, respectively. He started his career at AT\&T Bell Labs in 1986. Following Lucent Technologies spin off from AT\&T in 1996, he was with AT\&T Labs from 1996 to 2002. In 2002, he rejoined Bell Labs of Lucent Technologies. Since 2004, he has been the Tanaka Chair Professor of Internet technology at Imperial College. His research interests include radio resource allocation, MAC protocol, TCP/ IP protocol, mobility management, network architecture, real-time applications, and teletraffic issues for broadband wireless networks. $\mathrm{He}$ is also interested in a wide variety of wireless technologies, including 802.11, 802.16, and $3 G$ and future generation wireless networks. $\mathrm{He}$ received the Distinguished Member of Technical Staff Award from AT\&T Bell Labs in 1994, and was a corecipient of the 1997 Lanchester Prize Honorable Mention Award. He holds the Royal Society Wolfson Research Merit Award from 2004 to 2009. He is a fellow of the IEEE. $\mathrm{He}$ has published widely and acquired patents in many areas of communication networks. He has actively served on conference committees, including as the committee cochair for the Multiaccess, Mobility, and Teletraffic for Wireless Communications (MMT '98) and the committee vice-chair for the IEEE ICC 2002. He was a guest editor for the IEEE Journal on Selected Areas in Communications (JSAC) and the MONET Journal, and as an editor for the JSAC: Wireless Series. Currently, he is an editor for the IEEE Transactions on Communications and the IEEE Transactions on Wireless Communications.



Qiang Ni received the BEng, $\mathrm{MSc}$, and $\mathrm{PhD}$ degrees from Huazhong University of Science and Technology (HUST), Wuhan City, China in 1993, 1996, and 1999 respectively. He is currently a faculty member in the Electronic and Computer Engineering Division, School of Engineering and Design, Brunel University, West London, United Kingdom. From 2004 to 2005, he was a senior researcher at the Hamilton Institute, National University of Ireland, Maynooth. From 1999 to 2001, he was a postdoctoral research fellow in the multimedia and wireless communication laboratory, HUST, China. He visited and conducted research at the wireless and networking group of Microsoft Research Asia Lab during the year of 2000. From September 2001 until May 2004, he was a research staff member at the Planéte group of INRIA Sophia Antipolis, France. Since 2002, he has been active as a voting member at the IEEE 802.11 wireless LAN standard working group. He has served as Technical Program Committee (TPC) member/session chair for a number of international conferences on wireless communications and networking. His current research interests include communication protocol design, performance analysis, cross-layer optimizations and security issues for wireless networks, vertical handover and mobility management in mobile networks, and adaptive multimedia transmission over hybrid wired/ wireless networks. He has authored/coauthored more than 40 international journal/conference papers, book chapters, and standard drafts in this field. He is a member of the IEEE.

$\triangleright$ For more information on this or any other computing topic, please visit our Digital Library at www.computer.org/publications/dlib. 